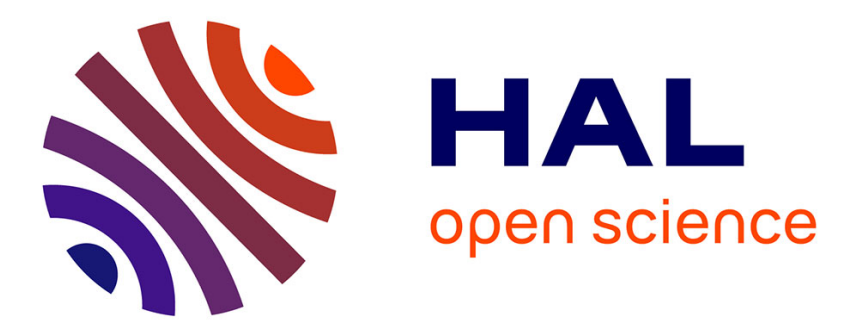

\title{
Réaménager l'espace: la régulation des conduites déviantes juvéniles dans le square Léon (Paris)
}

\author{
Ana Melo, Olivier Milhaud
}

\section{To cite this version:}

Ana Melo, Olivier Milhaud. Réaménager l'espace: la régulation des conduites déviantes juvéniles dans le square Léon (Paris). Desage Fabien; Sallée Nicolas; Duprez Dominique. Le contrôle des jeunes déviants, Les Presses de l'Université de Montréal, pp.218-233, 2015, 9782760634305. halshs-01374441

\section{HAL Id: halshs-01374441 \\ https://shs.hal.science/halshs-01374441}

Submitted on 28 May 2019

HAL is a multi-disciplinary open access archive for the deposit and dissemination of scientific research documents, whether they are published or not. The documents may come from teaching and research institutions in France or abroad, or from public or private research centers.
L'archive ouverte pluridisciplinaire HAL, est destinée au dépôt et à la diffusion de documents scientifiques de niveau recherche, publiés ou non, émanant des établissements d'enseignement et de recherche français ou étrangers, des laboratoires publics ou privés. 


\title{
Réaménager l'espace: la régulation des conduites déviantes juvéniles dans le square Léon (Paris)
}

\author{
Ana Maria Melo et Olivier Milhaud
}

Le réaménagement de l'espace urbain et l'urbanisme en général ne visent pas uniquement à ennoblir et à embellir la ville. Ils cherchent aussi à modifier le fonctionnement social, à influencer les relations et échanges qui se trament au cœur des villes. Les jeunes de classe populaire occupent une position tout à fait particulière, tant ils incarnent dans certains quartiers les tensions sociales entre générations, entre groupes sociaux, entre hommes et femmes, et même entre groupes ethnicisés. Par leur visibilité dans l'espace public et leur occupation d'espaces publics centraux, ils peuvent façonner l'image d'un quartier. C'est particulièrement le cas à la Goutte d'Or, un quartier du nord de Paris, où la présence de populations précaires et immigrées est particulièrement forte. Les profondes transformations du quartier ont servi plusieurs fonctions : participer d'une politique de peuplement (Desage, Morel Journel et Sala Pala, 2014), modifier l'offre commerciale, transformer l'image du quartier, mais aussi contrôler des pratiques déviantes. Le cas de la Goutte d'Or n'est pas choisi au hasard. Quartier emblématique de la Politique de la ville et de la recherche urbaine, la Goutte d'Or constitue un creuset d'immigration dans le nord de Paris, qui a connu de nombreuses opérations de rénovation urbaine ces dernières décennies. Parmi les ultimes bastions populaires de la capitale, il a été marqué historiquement par la présence d'une population peu aisée et d'origine étrangère, et par la précarité. Élus, artistes, journalistes, habitants présentent la Goutte d'Or comme une zone de non-droit ou, à l'opposé, comme un quartier "village» idéalisé et exotisé. Ces différentes représentations contribuent à façonner 
l'action publique sur son territoire. Au cœur de ce quartier se situe le square Léon, un axe de passage et un de ses rares espaces verts, également scène d'interventions d'aménagement et de régulation urbains qui s'insère dans le cadre du profond renouvellement du quartier. Le square constitue, dès lors, un point majeur de fixation, tant il incarne à la fois la vie sociale et les tensions du quartier, ainsi que les enjeux de son contrôle.

Ce chapitre se propose d'étudier comment le processus participatif de réaménagement de ce square depuis les années 2000 témoigne des contradictions entre volonté de réaménagement sécuritaire, aménagements paysagers visant à verdir le quartier, et présence maintenue du trafic de drogue. En grande partie cibles de l'action publique, les jeunes qui occupent le square n'ont pas su trouver leur place dans le processus "participatif» de réaménagement, à la différence des associations qui ont œuvré pour le verdissement du projet. Croiser les observations de terrain et un travail sur archives permet ainsi de mieux saisir les contra dictions du contrôle sociospatial des jeunes de classes populaires dans un site urbain. L'écart entre les objectifs et les résultats permet de confronter les intentions aménagistes, leur fétichisme spatial qui laisse à croire que changer l'espace change la société, le processus d'élabora tion d'un projet d'aménagement, et les résultats en matière de place laissée aux jeunes de classes populaires dans la construction de l'espace urbain et son occupation.

Dans un premier temps, nous tournerons notre regard sur la Goutte d'Or, "laboratoire » des sciences sociales et d'une action publique qui s'est faite très présente ces dernières décennies, pour ensuite centrer notre attention sur le square Léon et ses réaménagements avant de nous intéresser aux jeunes qui investissent ce square. Nous essaierons de montrer que ceuxci n'ont pas été de véritables acteurs du processus de réaménagement, mais qu'ils ont pu échapper en partie aux intentions aménagistes en détournant les usages du lieu rénové. D'où le paradoxe d'un espace paisible et vert, mais aussi d'une permanence du trafic de drogue, qui maintient une tension latente entre les divers acteurs de ce territoire. 


\section{Un quartier populaire emblématique}

La Goutte d'Or est de longue date le lieu d'installation de populations d'origines diverses. La construction des voies de chemin de fer et des gares parisiennes du nord et de l'est dans les années 18501860 stimule la construction de logements spéculatifs, attirant des classes populaires et fixant des ruraux, des provinciaux et des migrants européens. À partir du milieu du XX siècle, les populations maghrébines puis subsa hariennes marquent le territoire, respectivement au sud et au nord du quartier. Le quartier forme alors une véritable «centralité immigrée » (Toubon et Messamah, 1990).

Quartier phare de la "politique de la ville ${ }^{\text {[ }}$ ", il est la cible de nom breuses et profondes interventions sur le tissu urbain, sur le parc de logements et sur l'offre commerciale, influencées à la fois par les différentes images véhiculées par ce territoire, et par la pression de groupes locaux aux intérêts divers. Le fait que la Goutte d'Or reste un quartier populaire et d'immigration, longtemps marqué par la précarité, la vétusté et l'exiguïté des logements, renforce les discours publics sur le besoin de renouvellement urbain.

\section{La Goutte d'Or, données sociodémographiques}

Densément peuplée, la Goutte d'Or regroupe, en 2010, 32579 habitants répartis dans 16456 logements. Comme dans d'autres Zones urbaines sensibles (ZUS) de France ${ }^{\text {, }}$ les jeunes (32\% de moins de 25 ans), les familles monoparentales (22\%) et les familles nombreuses (17\%) sont surreprésentées (respectivement 28, 18 et 10 $\%$ à Paris). La proportion de cadres, significative (21\%), reste inférieure à la moyenne parisienne (32\%). À l'opposé, la proportion d'ouvriers est plus importante ( $15 \%$ contre $6 \%$ dans la capitale). Le taux de chômage est élevé ( $16 \%$ des actifs), corollaire d'une importante population sans diplôme ( $28 \%$ contre $13 \%$ à Paris).

1. Amorcée à la fin des années 1970, engagée dans les années 1980 et encore en cours, la Politique de la ville constitue l'ensemble des actions territorialisées, d'ordre social, économique et urbanistique, menées par l'État français en partenariat contractuel avec les collectivités locales et d'autres instances (organismes publics, bailleurs sociaux, milieux associatifs et économiques, etc.) en faveur de zones urbaines où la précarité est forte, visant à lutter contre leur dégradation et l'exclusion de leur population. Conçue comme discrimination positive territoriale, elle est aujourd'hui souvent considérée comme l'une des causes de la mise à l' écart spatiale de certaines populations.

2. Les ZUS sont des territoires urbains marqués par la précarité et délimités par les pouvoirs publics pour être la cible prioritaire de la Politique de la ville. Définies par la loi du 14 novembre 1996 relative à la mise en œuvre du pacte de relance pour la ville, elles sont actuellement 751 sur le territoire national. 
Le parc de logements reste marqué par l'étroitesse et la vétusté : 15\% des ménages ne disposent pas de salle de bain avec baignoire ou douche, $27 \%$ des logements ne comptent qu'une pièce et $51 \%$ font moins de $40 \mathrm{~m}^{\natural}$. Seuls $28 \%$ des résidences principales sont occupées par des propriétaires ( $33 \%$ dans la capitale) et avec $24 \%$ de locataires de HLM (17\% dans le $18^{\mathrm{e}}$ arrondissement et à Paris), la concentration du logement social est nette.

Enfin, la Goutte d'Or reste un quartier d'immigration : $27 \%$ de sa population est composée d'étrangers (19\% dans l'arrondissement, $15 \%$ à Paris et $6 \%$ en France métropolitaine).

Source des données (retravaillées par les auteurs): Recensement de la population pour l'année 2010 (INSEE - Institut national de la statistique et des études économiques, 2013).

Les représentations artistiques, médiatiques, politiques et académiques ont proliféré depuis le roman L'Assommoir d'Émile Zola (1876). Le quartier - emblématique des luttes urbaines des années 1980 et d'un Paris populaire et immigré en voie de disparition/reconfiguration aujourd'hui - est devenu un laboratoire de la ville en mutation, tant pour les politiques publiques que pour les chercheurs en sciences sociales qui l'ont largement exploré (Toubon et Messamah, 1990 ; Bacqué et Fijalkow, 2006 ; Pinçon et Pinçon-Charlot, 2001 ; Chabrol, 2011).

La presse nationale, quant à elle, peut se faire l'écho de l'invasion des espaces publics pour les prières du vendredi, faute de mosquées suffisantes pendant une longue période, de l'insécurité qui y règne, ou bien vanter "l'un des derniers quartiers parisiens pour réaliser de bonnes affaires immobilières " ou se plonger dans une ambiance " africaine ». Alors que les journaux gratuits des pouvoirs publics promeuvent les opérations urbaines et les mutations physiques du quartier à coup de démolitions/reconstructions, la presse de quartier, particulièrement vivante, dénonce la brutalité des changements (Palumbo, 2009).

Un discours sécuritaire accompagnant ces opérations radicales de rénovation/réhabilitation domine aujourd'hui les représentations des autorités municipales. Les politiques publiques tentent de "désethniciser» le territoire par une diversification commerciale, avec même la constitutiond'unerue delamode,s'éloignantd'une offrecommerciale perçue comme trop africaine, trop liée à des populations migrantes fraîchement arrivées. Ces tentatives de limiter la prééminence de commerces africains s'accompagnent d'actions de valorisation culturelle et sociale, autour de l'imaginaire exotique qui entoure la Goutte d'Or. Quant auxassociations locales, à vocation sociale ou 
culturelle, diverses et nombreuses dans l'intervention auprès des jeunes, des migrants ou des toxicomanes, elles s'opposent toutes à la stigmatisation du quartier, mais ne sont pas mues par les mêmes logiques. Dès les années 1980, "Paris Goutte d'Or " (portée par des résidents impliqués dans le tissu associatif local) tente de minimiser l'ampleur des interventions urbaines pour s'assurer que la population résidente demeure sur place. D’autres cherchent plutôt à éliminer les nuisances du quartier (le trafic de drogue était particulièrement visé par l'association Droit au calme dans les années 2000, par exemple), ou à s'opposer aux opérations de transformation urbaine au nom du "patrimoine » du quartier (l'association Cavé Goutte d'Or qui milite pour préserver le bâti faubourien y compris modeste, souhaitant allier logement social et esthétique urbaine). Ces différents groupes influencent l'action des pouvoirs publics, et c'est dans ce cadre qu'il faut comprendre l'intervention dans le square Léon.

\section{Une action publique soutenue}

À l'image de ce que rapportent les travaux sur les banlieues françaises (Kokoreff, 2003 ; Lapeyronnie, 2008), les représentations de la Goutte d'Or oscillent entre celle d'un quartier immigré et dangereux et celle d'un quartier "village », multiculturel et idéalisé. Cette dichotomie «rencontre la diversité des représentations habitantes, qui, en retour, pèsent lourdement sur les orientations en apparence contradictoires du pouvoirmunicipal» (Bacqué et Fijalkow, 2006, p. 71).

Il faut dire que la Mairie a su profiter de l'inscription de la Goutte d'Or comme quartier emblématique de la Politique de la ville française. Elle fut ainsi l'un des premiers quartiers classés «îlot sensible», en 1983, ancêtre de la Zone urbaine sensible, qu'elle devint dès 1996. Ces dispositifs, maintenus jusqu'à maintenant, témoignent de 30 années d'interventions successives dans le quartier. En 2012, l'action publique a maintenu son caractère " exceptionnel » en faisant de la zone Barbès/ ChâteauRouge/Goutte d'Or une des Zones de sécurité prioritaire (ZSP) sur le territoire national. De fait, la Goutte d'Or a été un lieu d'expérimentation de nombreux dispositifs, les démolitions/reconstructions s'accompagnant de diverses politiques sociales et de sécurité ${ }^{\boxplus}$.

3. Parmi les nombreuses interventions qui ont eu lieu à la Goutte d'Or, les opérations de requalification urbaine dans le secteur sud du quartier, à partir de 1983, et dans la zone de Château-Rouge (dans le cadre d'un Plan d'urgence), 
Cette focalisation des pouvoirs publics sur la Goutte d'Or s'explique certes par l'importance de la précarité urbaine et sociale du quartier (drogue, délinquance, prostitution), mais aussi par l'audience politique du $18^{\mathrm{e}}$ arrondissement, enjeu considérable dans la conquête de la Mairie de Paris, et où des figures remarquables de la vie politique nationale (deux anciens premiers ministres du pays, Alain Juppé et Lionel Jospin) ont œuvré. La Goutte d'Or joue un rôle important dans la revendication d'un bilan politique "social», car c'est un des lieux de Paris où la construction de logements sociaux semble la plus légitime à mettre en œuvre, tout en étant facilitée par des prix de l'immobilier moins élevés qu'ailleurs.

Ces politiques ont représenté une chance pour la Goutte d'Or, qui a bénéficié de nombreux financements nationaux pour des programmes, projets et opérations urbains. Cependant, le stigmate lié au label ZUS et aux opérations de dramatisation de la situation de délinquance demeure. De plus, l'expulsion progressive des classes populaires a été réalisée par des opérations urbaines, dans lesquelles l'injonction à la mixité sociale apparaît comme un leurre favorable à la gentrification du quartier ${ }^{\natural}$. Si le parc locatif d'habitations à loyer modéré (HLM) s'est développé depuis 1990, les opérations ont été menées brutalement (beaucoup d'expulsions et de démolitions), dans un but explicite de liquidation de I'héritage immigré, notamment algérien. La normalisation urbaine (façades, commerces) apparaît comme un préalable à l'installation de nouvelles populations, à la faveur d'augmentations de loyer trop sensibles pour les plus modestes.

depuis le début des années 2000, doivent être soulignées. Une grande partie de ces territoires a été démolie et des équipements publics et des immeubles neufs à usage social ont vu le jour. Des dispositifs d'incitation à la réhabilitation du parc immobilier privé ont été également mis en place, comme les diverses Opérations programmées d'amélioration de l'habitat (OPAH). Dans le cadre du Contrat urbain de cohésion sociale (CUCS), entre 2007 et 2010, et des «projets de territoire » (pour les secteurs Amiraux-Simplon et Goutte d'Or), à partir de 2010, par exemple, outre la poursuite des interventions physiques, des actions sociales et de sécurité ont été développées, parmi lesquelles plusieurs destinées aux jeunes (accompagnement scolaire; offre de loisirs péri et extrascolaires; actions articulant prévention, médiation et animation dans l'espace public; expérimentation d'un espace de médiation parents-adolescents; aide à la constitution d'associations portées par des jeunes...).

4. La tendance à la gentrification peut être vérifiée par l'analyse de l'évolution sociodémographique du quartier entre 1999 et 2007 : on constate une augmentation de 6 points de la part des cadres (de $11 \%$ à $17 \%$ ) et de 3 points des professions intermédiaires (de12\% à 15\%)(Chabrol, 2011). 
Dans un tel contexte, la participation des habitants, qui fut un maître mot de la Politique de la ville dans les années 1980, n'allait pas de soi. Certaines associations ont bien accompagné le processus, mais elles ont surtout reflété la prééminence, dans cette représentation symbolique, des habitants dotés de davantage de capital social et financier (Bacqué et Fijalkow, 2006). Les enjeux de l'opération du square Léon laissent bien transparaître ces rapports de force internes aux quartiers où les associations les plus légitimes ne donnent pas forcément toute leur place aux jeunes de classes populaires qui occupaient pourtant les lieux.

\section{Le square Léon, place centrale du quartier à réaménager}

Véritable place centrale et point le plus haut du quartier, le square Léon constitue autant un point de jonction entre la Goutte d'Or sud maghrébine et la Goutte d'Or nord noire-africaine qu'un véritable carrefour entre les axes structurants du territoire. De fait, l'emplacement était initialement un passage (le passage Léon, du nom du propriétaire du terrain sur lequel la voie a été ouverte). Un obus tombé lors de la Seconde Guerre mondiale l'a transformé en terrain vague. La démolition d'immeubles insalubres permit en 1973 la constitution d'un premier "square du passage Léon ».

Un premier réaménagement du square en 1990 permet d'en faire un lieu de rassemblement pour les mères et leurs enfants, les adolescents, et les vieux jouant aux dames. Toutefois, les scènes d'injection restent fréquentes le jour et l'occupation des lieux bruyante la nuit. Les tessons de bouteillejonchentlesol,alorsmême qu'ils'agitd'un desrares espaces « verts» du quartier. En 1995, le nouveau maire de Paris, Jean Tibéri (RPR, droite), soutient que la Goutte d'Or a trop reçu du temps de la mandature précédente et coupe les subventions. Dans le square, la situation se dégrade. Trafic, vandalisme, combats de pitbulls, jeux de ballon au cœur de la nuit et manque d'entretien du square poussent les riverains à la mobilisation. En 1996, l'adjointe au maire de Paris entend « reconquérir l'espace», notamment en fermant le square par une grille, qui serait - à terme - verrouillée la nuit. Les jeunes hommes habitués du square sont clairement visés par la démarche. La grille est installée en 1998, son portail rapidement cassé l'année d’après. En 1999, Souleymane Fofana, 19 ans, est abattu dans le square en pleine nuit, sans qu'on sache très bien s'il s'agit d'une bavure policière de la Brigade anti-criminalité (BAC) 
présente sur les lieux au moment du drame ou d'un suicide du jeune (tué avecl'armequ'il détenait). Régulièrement, les armoires EDF qui assurent l'éclairage public sont vandalisées, plongeant l'espace et les rues adjacentes dans l'obscurité, ce qui favoriserait les trafics. En 2000, l'arrivée au ministère de l'Intérieur de Daniel Vaillant (maire de l'arrondissement tenu depuis 1995 par le PS) et une campagne médiatique dénonçant la «zone de non-droit » de la Goutte d'Or permettent d'accroître la présence policière dans le quartier et les opérations « coups de poing ». Cela aboutit à une plus grande mobilité du trafic de drogue, qui se replie souvent sur le square Léon, d'autant plus qu'il manque de gardiens.

À la demande d'associations et de parents de la Goutte d'Or, une première réunion publique sur le devenir du square se tient fin 2001. Elle fait rapidement apparaître les divergences entre les attentes des riverains qui souhaitent jouir d'un espace vert digne de ce nom, propre et entretenu, et la reconnaissance que ce square est, de fait, un lieu de sociabilité important pour des populations d'âges divers du quartier, un passage public crucial dans le fonctionnement de la Goutte d'Or, une aire de jeux pour enfants et adolescents, et un espace pour des usagers toxicomanes ou jugés trop bruyants la nuit. Lássociation «Droit au calme " (composée d'habitants plutôt aisés arrivés récemment dans le quartier et fondée en 2000 pour lutter contre "le marché des voleurs" qui revendent de la maroquinerie dans la rue voisine de Panama) souhaite la fermeture nocturne du square à l'instar des autres squares parisiens. La Mairie profite alors du projet de mise en conformité des aires de jeux du square pour en repenser l'organisation et le fonctionnement global. Elle opte pour une large concertation, entre juillet et décembre 2003, dans le cadre d'ateliers avec des usagers, des riverains et les services techniques de la mairie.

\section{Les jeunes, cibles d'un projet qui leur échappe}

Dans ce processus d'élaboration du nouvel aménagement, l'enjeu sécuritaire concurrence les objectifs de verdissement, marginalisant de fait le point de vue des jeunes qui n'ont pas su faire entendre leur voix. La synthèse des échanges entre certains usagers, des riverains impliqués et le groupe technique de la Ville de Paris peut être lue dans un Mémento de programmation d'une dizaine de pages, élaboré par la Mairie de Paris à l'issue des ateliers (Ville de Paris, 2004). Il faut bien sûr la lire entre les lignes. Ce qui ressort alors, à travers 
les «intentions de résolution" des problèmes, renvoie à la définition - par un traitement extrêmement fin des espaces - d'un lieu public ouvert à tous, fait d'une coexistence harmonieuse entre les groupes, dans un espace vert de qualité. Les jeunes ethnicisés y auraient une place bien définie, très précisément délimitée, sans bouleverser de fond en comble l'organisation initiale du square.

La synthèse des échanges incite ainsi à «s'appuyer sur les qualités d'usage et [...] les qualités de paysage » (ibid.) de l'existant, comme conserver les bancs sous les platanes qui longent au sud l'extérieur du square auxquels on prête la capacité de « réguler des pratiques plus bruyantes et potentiellement dérangeantes " (ibid.). La suggestion est aussi faite de rendre certaines rues adjacentes non circulantes et d'élargir les trottoirs. En somme, il s'agit d'inciter à un usage paisible du square avant même d'y avoir pénétré. Au cœur du square, les jeunes apparaissent plusieurs fois comme l'une des cibles principales du projet de réaménagement. On comprend vite qu'il s'agit plus précisément de garçons adolescents, dont la présence et les activités sont associées à une nuisance sonore. II faudrait par exemple "limiter le bruit lié au jardin" (ibid.) en jouant particulièrement sur le sol en béton du terrain de football et remplacer les grilles métalliques qui l'entourent par un revêtement synthétique et des filets. De même, il s'agit " d'empêcher le débordement des pratiques de foot sur tout l'espace du jardin et assurer la protection des pelouses " (ibid.), en créant un espace central très vallonné ou parsemé de bosquets de fleurs ou de petits arceaux. Le Mémento appelle aussi à développer l'offre de jeux par tranche d'âge: 2-6 ans, 6-12 ans, et enfin pour les 13-20 ans est suggérée la création d'«un espace foot - basket rénové, des bancs, un point d'eau propre», car il faut "améliorer les conditions d'exercice d'une activité sportive pour les jeunes " (ibid.). Mais pour " permettre aux uns et aux autres de trouver leur place sans perturber celle des autres " (ibid.), il s'agit bien sûr de distinguer, matériellement, visuellement, ces espaces. Dès lors, l'objectif est de diversifier les espaces tout en les séparant («assurer des transitions physiques et visuelles entre des espaces aux activités peu compatibles " [ibid.]).

La mixité des générations et des sexes est encouragée à l'échelle du square dans son entier, mais dissuadée dans les sous-espaces, dont les usages sont fonctionnalisés. L'espace jeux de dames, pour les anciens, doit par exemple être amélioré en ajoutant des tables et des tabourets. 
Il faut aussi «féminiser les pratiques du square» (ibid., p. 9) en disposant des espaces pour les petits et moyens (jusqu'à 12 ans), entourés de bancs qui seraient pour certains en cercle "pour conforter ce lieu de rendez-vous des mamans" (ibid., p. 9). Les femmes sont donc essentiellement appréhendées comme de jeunes mères, dont il faudrait garantir la présence dans le square sans les mêler aux adolescents: d'où la proposition d'«implanter des bancs, dos aux terrains de sports permettant aux mamans de surveiller leurs enfants, sans être trop confrontées aux jeunes ou aux squatters » (ibid., p. 9). La séparation des groupes est renforcée par une multiplication des traversées possibles: cela permettra de "gérer au mieux certaines captations de l'espace par des groupes auxquels on ne souhaite pas se confronter " (ibid., p. 5). Tout un travail est encouragé sur la visibilité des lieux. II faut éviter les arbustes propices aux cachettes et les recoins, "ouvrir largement les vues sur le jardin » depuis les rues avoisinantes " afin de favoriser le contrôle sur ce qui se passe à l'intérieur depuis les rues» (ibid., p. 5). L'éclairage nocturne doit venir en renfort. II sera robuste et sur "des implantations difficilement accessibles aux dégradations » (ibid., p. 5). Supprimer tout mur en béton brut propice aux tags et végétaliser les parties verticales jusqu'à trois mètres de haut éviterait des appropriations graphiques perçues comme des détériorations du lieu. Dans l'idée de faire du square un espace appropriable par tous, un large espace central ouvert (et vallonné pour éviter les parties de foot) doit être créé pour y tenir des manifestations comme la fête de la Goutte d'Or, où se retrouverait toute la population du quartier.

Les jardiniers doivent être libérés du travail de nettoyage pour investir qualitativement l'espace (fleurs et plantations), " maximiser la présence végétale dans le square et autour" (ibid., p. 10), définir des ambiances végétales différentes.

Il faudrait " développer la gestion et le gardiennage » (ibid., p. 3), en disposant d'au moins un gardien à plein temps avec une loge à vue panoptique, et "doubler le poste par un éducateur susceptible de transmettre les nouvelles règles du partage de l'espace (aux jeunes footballeurs notamment, mais également aux plus petits) » (ibid., p. 3). Dans un courrier adressé à la Mairie, I'association de quartier Paris-Goutte d'Or insiste sur l'importance de limiter les investissements (en ne remplaçant pas la grille par exemple, érigée à la fin des années 1990) afin d'augmenter les moyens humains de surveillance et d'entretien. La nécessité 
d'éliminer seringues, déjections canines, tessons de bouteille avant l'arrivée des enfants (de 7 à 9 heures) est rappelée.

Les recommandations du Mémento de programmation donnent l'impression d'une grande cohérence et d'un travail très fin sur les espaces. Pourtant, les incompatibilités sont inévitables. II est par exemple proposé de "disposer d'un éclairage différent pour les parties hors des passages, [...] plus lié au paysagement, mais suffisant pour éviter toutes zones d'ombre propices aux trafics, aux "promenades de chiens le soir", sans pour autant favoriser les regroupements nocturnes aux beaux jours " (ibid., p. 5). La dimension sécuritaire ne se marie pas facilement avec l'aspect esthétique du square, qui doit être également un espace vert, fleuri et boisé. Les arbustes sont propices au verdissement, mais aussi aux cachettes... Les aménageurs doivent composer avec ces contradictions.

\section{Le réaménagement d'un espace vert}

Le résultat de la consultation aboutit à quantité de choix d'aménagement et de fonctionnement en faveur du verdissement du square, avec presque 190 arbres, 5000 arbustes et 1500 plantes vivaces, et une grande pelouse centrale. L'appropriation par tous devrait être assurée et le caractère bucolique devrait diversifier la fréquentation du lieu. Au nord-ouest se trouveront des terrains de foot et de basket ainsi que des tables de ping-pong pour les jeunes, et à l'est la grande pelouse dans la partie nord et des jeux pour les petits dans la partie sud, des arbres tout autour. Les personnes âgées conserveront la "placette des échecs", au sud-ouest, qui se trouve pile sur le passage central, munie de tables plus nombreuses. Avec le local du gardien et les toilettes publiques entre les jeux des petits au sud-est et la placette des échecs au sud-ouest, l'entrée sud devrait logiquement échapper au contrôle des jeunes. Les espaces seront bien délimités, notamment pour les jeux des tout petits, enclos par des grilles basses doublées de végétation buissonnante. Six entrées dans le square et le tracé de sentiers en terre battue permettront des itinéraires différents du seul passage Léon. L'éclairage sera renforcé et sécurisé. Un gardien partagera sa journée entre le square Léon et le square voisin. Mais le réaménagement et le verdissement ont coûté cher, et il n'est pas prévu de poste d'éducateur. II n'y aurait personne la nuit. En 2006, la Ville passe une convention avec Nike pour financer le gazon synthétique du terrain de foot (30 000 euros), moins bruyant, inauguré 
figure 1

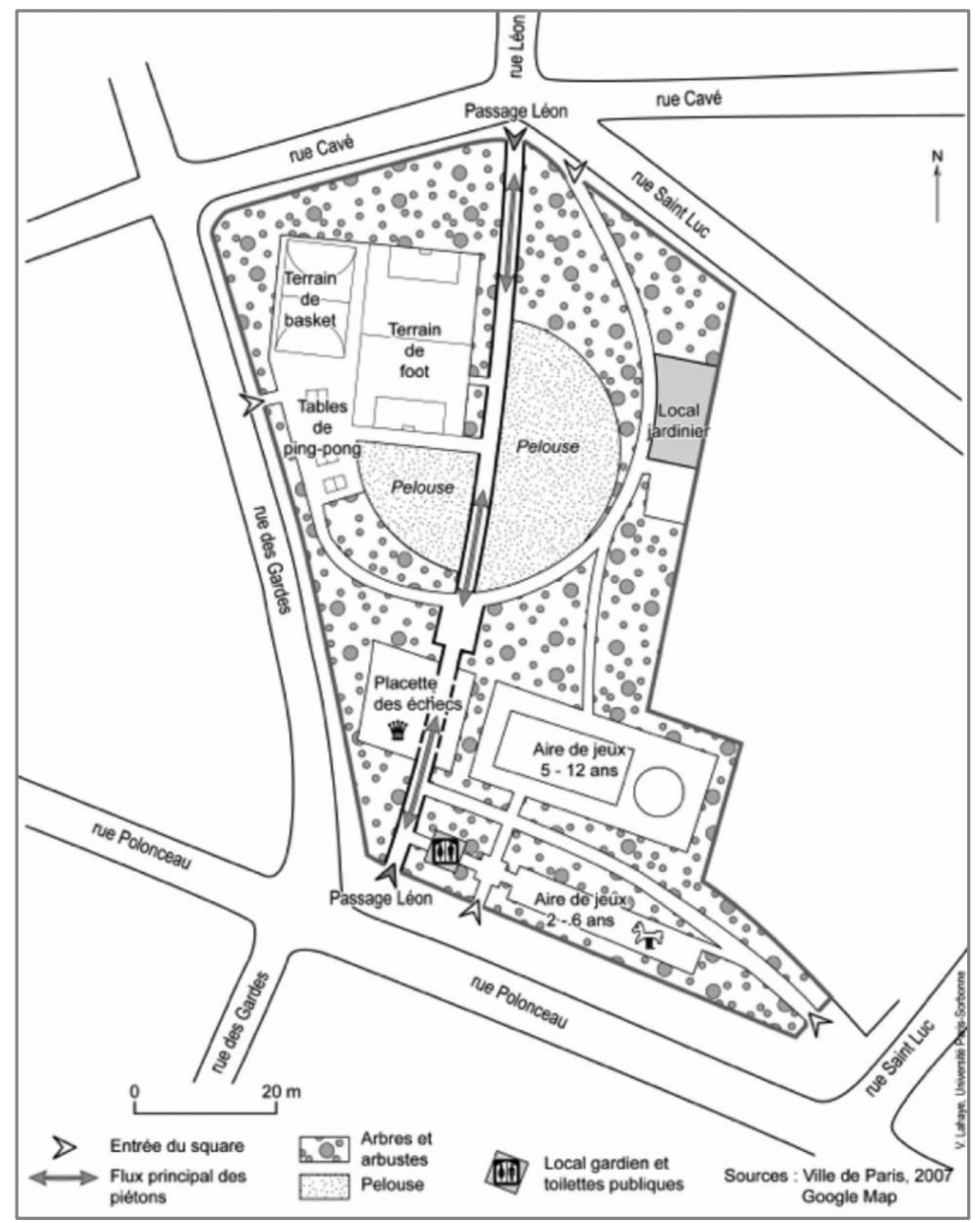

en présence du footballeur Lilian Thuram, venu rencontrer I'association des Enfants de la Goutte d'Or (dédiée notamment au soutien scolaire des enfants du quartier) qui avait milité pour cette solution face au manque de financements publics.

La brochure municipale distribuée aux habitants à la réouverture du square au printemps 2007 insiste résolument sur la dimension espace vert, au point d'en gommer tout enjeu de sécurité : «Le square Léon... un nouveau jardin dans votre quartier [...] Cet espace vert a été conçu pour que les habitants du quartier puissent bénéficier d'un coin de verdure et de détente à côté de chez eux » (Ville de Paris, 2007). Le mensuel du quartier, Le [?] $d u$ mois, titre en avril 2007 «Un square Léon tout neuf pour le printemps ", en mai "Square Léon: mention très bien mais... » et, dès le mois de juin, «Fermer le square Léon la nuit? ». Entre 
l'ouverture le 6 avril, et le 14 mai, on compte 27000 euros de dégâts. Les nuisances sonores se poursuivent, malgré les réaménagements.

\section{Les pratiques spatiales et les détournements d'usage}

En novembre 2008, décision est prise de fermer le square la nuit, ce que réclamaient les riverains. Les dégradations s'élevaient à 100000 euros (pour un budget de rénovation de 1300 000). 177 agressions verbales ou physiques sur les agents de surveillance ou d'entretien avaient été recensées. Surtout, la police n'arrivait plus à contrôler le trafic dans le square la nuit. l'éclairage du passage ne sera plus à quatre mètres de haut, mais à neuf (trop d'ampoules brisées par des cailloux), des portillons tournants empêcheront les intrusions de vélos, de scooters et de motos, et la police s'engage à surveiller les abords du square la nuit, pour éviter un simple déplacement du trafic. La plupart des associations ne soutiennent pas totalement le projet de fermeture, préférant une poursuite de la concertation. En février 2009, la fermeture entre en vigueur à partir de $23 \mathrm{~h} 30$ (ce qui en fera le square à la fermeture la plus tardive de Paris), à l'issue d'une rencontre houleuse organisée par la mairie d'arrondissement, où les jeunes venus à la réunion (à la différence des associations qui n'ont pas voulu s'y rendre) se sentent clairement visés par la mesure. L'observation des pratiques spatiales dans le square Léon, à des dates et à des périodes de l'année différentes, s'avère très instructive. Déambuler dans le square comme un passant du quartier, s'installer sur un des bancs, se rendre à une des festivités organisées dans le square, ou enfin observer l'espace depuis les rues avoisinantes, permet de mieux comprendre son fonctionnement au quotidien. Il est assurément resté un espace central du quartier. L'été, en particulier, il est très fréquemment investi par les associations pour des activités d'arts plastiques, une bibliothèque hors les murs qui s'installe avec livres et bibliothécaires sur la pelouse, ou encore des jeux en plein air. II n'empêche, les membres des associations sont souvent interpellés par certains jeunes adultes habitués du square qui leur demandent: "Qu'est-ce que vous faites chez nous?" L'espace jeux des petits est bien investi par les mères et les enfants d'origine africaine et asiatique en fin de journée, les mercredis et les fins de semaine. Les échanges entre mères racisées de différentes origines sont rares néanmoins. La placette des échecs est occupée la majeure partie de la journée par des hommes âgés maghrébins, qui jouent aux dames. L’allée centrale du passage traverse la grande pelouse vallonnée. Celle-ci est bien 
évidemment investie les jours de fête du quartier, mais la plupart du temps elle est plutôt vide, avec des adolescents de 16-20 ans, d'origine africaine, assis de part et d'autre de l'allée à proximité de l'entrée du terrain de foot. Ils n'hésitent pas à jauger la silhouette des jeunes femmes passant par là pour relier le nord et le sud du quartier et à dévisager les habitants qui passent. De fait, toute personne traversant le square doit passer parmi ces jeunes adultes, à moins d'emprunter l'itinéraire est - en partie sous les arbres - qui longe le local des jardiniers et où se déroule une partie du trafic, ou bien d'éviter le square en passant par la rue des Gardes, à l'ouest, où sont postés des jeunes "guetteurs", surveillant la venue de la police.

Le trafic de drogue se poursuit au vu et au su de tous, mais dans un calme relatif. La rencontre a lieu inévitablement sur l'allée centrale, où les jeunes hommes contrôlent, de fait, le passage. La transaction peut se faire très rapidement au beau milieu du square donc, mais en étant sous l'angle de vision des très hautes caméras de sécurité panoramiques à l'entrée du jardin. Le plus souvent, après un premier échange verbal au milieu du passage, vendeurs et acheteurs se dirigent vers le local (souvent fermé) du jardinier côté est, à l'abri des arbres et des caméras, où la transaction s'opère.

Les arbustes abondants dans le square offrent un nombre incalculable de cachettes possibles pour la marchandise. Qui plus est, l'arrivée de la police est bien souvent annoncée par les guetteurs situés rue des Gardes. Quand un car de CRS se stationne le long du square, on peut voir l'ensemble des jeunes hommes disputer une partie de football endiablée, massés dans un terrain de football bien trop petit pour pouvoir raisonnablement jouer au ballon, mais où le nombre et la compacité des joueurs dissuadent toute incursion de la police. Le grillage entourant le terrain évite certes que les ballons sortent du square, mais il se transforme en délimitation d'un territoire jeune et masculin difficilement pénétrable pour les forces de l'ordre. Celles-ci préfèrent le plus souvent rester à l'extérieur du square, ou bien y pénétrer le soir pour aider le gardien à faire sortir les jeunes et fermer les grilles. C'est le moment le plus tendu de la journée, les jeunes essayant de retarder l'heure, gardiens et forces de l'ordre tâchant d'envenimer le moins possible la situation. Les agressions ont baissé, les nuisances sonores tardives nettement moins. 
Les jeunes étaient parmi les principaux occupants du square et le processus de réaménagement se présentait comme une ouverture aux souhaits des usagers. Malgré cela, ceux-ci n'ont pas su trouver leur place, à la différence des associations qui ont su œuvrer pour le verdissement du projet et doter ainsi le quartier d'un véritable espace vert. Sans doute les pouvoirs publics y ont-ils vu un moyen de changer l'image de la Goutte d'Or, d'attirer plus de monde sur cette place centrale du quartier, et ainsi de reconquérir l'espace tout en laissant des espaces de sport pour les jeunes. Cependant, le contrôle spatiotemporel de ceux-ci - théoriquement cantonnés dans certains espaces - n'empêche nullement des détournements d'usage propices au trafic de drogue. Comme si le verdissement du square s'était paradoxalement révélé plus favorable au trafic, en lui offrant une certaine paix (et invisibilité), éloignant la consommation, qui ne se fait plus nécessairement sur place.

À première vue, le gros travail de réaménagement peut être perçu comme un succès en matière de participation au changement d'image du quartier (reconstructions de logements, nouveaux commerces, nouvel espace vert) et de fréquentation du square, ce qui ne peut que participer au processus de gentrification en cours. En réalité, les populations y cohabitent, chacune dans ses sous-espaces (les vieux sur la placette des échecs, les jeunes mères près des jeux des petits, les jeunes ethnicisés côté terrains de sport et sur toute la partie nord du square), ou s'y croisent sans forcément interagir. Et le trafic continue. Lappropriation du square par les jeunes ná pas été éradiquée, mais a été redéfinie plus subtilement. Laménagement d'un espace vert plus que dún espace sûr a finalement pacifié les relations, tout en masquant les luttes pour le contrôle du quartier. Les associations, si présentes à la Goutte d'Or, font du square un lieu central pour toutes sortes de manifestations, mais de manière très ponctuelle, alors que les jeunes l'occupent de façon plus soutenue. Comparés aux objectifs du Mémento de programmation, on ne peut pas parler d'un square sûr et appropriable par tous, mais plutôt d'un espace vert qui segmente les groupes et multiplie les caches possibles.

Cette situation remet en question plus largement le spatialisme des aménageurs qui, en se limitant à une action sur l'espace physique/matériel, espèrent changer radicalement les relations sociales, alors que les usages qu'ils prescrivent sur leurs plans sont bien souvent détournés. De plus, les processus de 
participation qui apparaissent comme des impératifs politiques de la production des espaces publics contemporains tendent à accroître les contradictions entre les ambitions des groupes qui savent y participer et les ambitions initiales du projet. Le paradoxe du réaménagement du square Léon est qu'il aura abouti à un espace très peu sécuritaire mais paisible, laissant aux jeunes le contrôle de fait du passage et des espaces de pratique sur une bonne moitié du square, tout en consacrant leur marginalisation dans le processus politique de participation et la gentrification du quartier. 